\title{
Stability and Convergence of Spectral Methods for Hyperbolic Initial-Boundary Value Problems
}

\author{
By P. Dutt
}

\begin{abstract}
In this paper we present a modified version of the pseudospectral method for solving initial-boundary value systems of hyperbolic partial differential equations. We are able to avoid problems of instability by regularizing the boundary conditions. We prove the stability and convergence of our proposed scheme and obtain error estimates.
\end{abstract}

1. Introduction. In this paper we study the stability and convergence of spectral methods for the approximation of initial-boundary value hyperbolic systems with constant coefficients.

This problem has been studied by Gottlieb, Lustman and Tadmor [1], [2] under the assumption that the boundary conditions are dissipative. We prove that a modified version of the numerical scheme they have proposed is stable and converges to the true solution of the hyperbolic initial-boundary value problem (IBVP), without any assumption of dissipativity on the boundary conditions. Our treatment closely follows their approach.

Definitions. Consider the first-order hyperbolic system of partial differential equations

$$
\frac{\partial \mathbf{u}}{\partial t}=A \frac{\partial \mathbf{u}}{\partial x}, \quad-1 \leq x \leq 1, t \geq 0 .
$$

Here, $\mathbf{u}=\mathbf{u}(x, t)=\left(u^{1}, \ldots, u^{n}\right)^{T}$ is the vector of unknowns, and $A$ is a fixed $n \times n$ coefficient matrix.

Since by hyperbolicity, $A$ is similar to a real diagonal matrix, we may assume without loss of generality that it is diagonal:

$$
A=\left[\begin{array}{cc}
A^{\mathrm{I}} & 0 \\
0 & A^{\mathrm{II}}
\end{array}\right], A^{\mathrm{I}}=\left[\begin{array}{ccc}
a_{1} & & \\
& \ddots & \\
& & a_{l}
\end{array}\right]<0, \quad A^{\mathrm{II}}=\left[\begin{array}{ccc}
a_{l+1} & & \\
& \ddots & \\
& & a_{n}
\end{array}\right]>0
$$

The solution of this system is uniquely determined if we prescribe initial conditions

$$
\mathbf{u}(x, 0)=\mathbf{f}(x), \quad-1 \leq x \leq 1,
$$

and boundary conditions

$$
\begin{aligned}
& \mathbf{u}^{\mathrm{I}}(-1, t)=L \mathbf{u}^{\mathrm{II}}(-1, t)+\mathbf{g}^{\mathrm{I}}(t), \quad t \geq 0 . \\
& \mathbf{u}^{\mathrm{II}}(1, t)=R \mathbf{u}^{\mathrm{I}}(1, t)+\mathbf{g}^{\mathrm{II}}(t),
\end{aligned}
$$

Received September 22, 1988; revised December 7, 1988.

1980 Mathematics Subject Classification (1985 Revision). Primary 65M10, 65M15, 65N35.

Key words and phrases. Initial-boundary value problems, regularization, convolution, stability, convergence. 
In these formulas,

$$
\mathbf{g}=\mathbf{g}(t)=\left(\mathbf{g}^{\mathrm{I}}(t), \mathbf{g}^{\mathrm{II}}(t)\right)^{T}
$$

is a given $n$-vector, and

$$
\mathbf{u}^{\mathrm{I}}=\left(u^{1}, \ldots, u^{l}\right)^{T}, \quad \mathbf{u}^{\mathrm{II}}=\left(u^{l+1}, \ldots, u^{n}\right)^{T}
$$

is a partition of $\mathbf{u}$ into its inflow and outflow components - corresponding to the partition of $A$ in (1.1b) - while $L$ and $R$ are constant reflection matrices of order $l \times(n-l)$ and $(n-l) \times l$, respectively.

The system (1.1a)-(1.1e) is a well-posed problem in the sense described by Kreiss in [4].

We study the pseudospectral discretization of (1.1). In any such approximation, one seeks a vector of $N$ th degree polynomials

$$
\mathbf{z} \equiv \mathbf{z}_{N}(x, t)=\left(z_{N}^{1}(x, t), \ldots, z_{N}^{n}(x, t)\right)^{T}
$$

such that

$$
\frac{\partial \mathbf{z}}{\partial t}=A \frac{\partial \mathbf{z}}{\partial x}+Q(x) \boldsymbol{\gamma}
$$

where $\gamma=\left(\gamma^{\mathrm{I}}, \gamma^{\mathrm{II}}\right)^{T}$ is an $n$-vector.

In the pseudospectral Chebyshev method, we collocate at the interior extrema of $T_{N+1}(x)$, yielding

$$
Q(x)=T_{N+1}^{\prime}(x) \cdot I,
$$

where $I$ is the $n \times n$ identity matrix and $T_{k}(x)$ is the Chebyshev polynomial of degree $k$. In [1], Gottlieb, Lustman and Tadmor proved that the spectral approximation (1.2a) with zero initial conditions and subject to the boundary conditions

$$
\begin{aligned}
& \mathbf{z}^{\mathrm{I}}(-1, t)=L \mathbf{z}^{\mathrm{II}}(-1, t)+\mathbf{g}^{\mathrm{I}}(t), \quad t \geq 0, \\
& \mathbf{z}^{\mathrm{II}}(1, t)=R \mathbf{z}^{\mathrm{I}}(1, t)+\mathbf{g}^{\mathrm{II}}(t),
\end{aligned}
$$

is stable provided that the boundary operators satisfy the dissipativity condition

$$
|R||L|<1 .
$$

They further showed that if we prescribe initial data

$$
\mathbf{z}(x, 0)=E \mathbf{u}(x, 0)=E \mathbf{f}(x),
$$

where $E$ is a projection operator, then $\mathbf{z}_{N}(x, t)$ converges to the solution $\mathbf{u}(x, t)$ of (1.1) as $N \rightarrow \infty$.

Here and throughout the paper we denote by $|\mathbf{v}|$ the Euclidean norm of a vector $\mathbf{v}$; similarly, $|A|=\sup |A \mathbf{v}| /|\mathbf{v}|$.

If condition (1.2c) is violated, however, the pseudospectral approximation (1.2) may be unstable.

In the method presented in this paper we collocate at the same points; however, we are able to avoid problems of instability by working with a regularized version of the boundary conditions. Let $\mathbf{v}$ and $\tau$ be $n$-vectors,

$$
\mathbf{v}=\left(\mathbf{v}^{\mathrm{I}}, \mathbf{v}^{\mathrm{II}}\right)^{T}, \quad \tau=\left(\tau^{\mathrm{I}}, \tau^{\mathrm{II}}\right)^{T},
$$

as in the previous discussion. 
We first collocate the partial differential equation at the $N$ points

$$
x_{i}=\cos (i \pi /(N+1)), \quad i=1, \ldots, N,
$$

to obtain the spectral approximation

$$
\begin{aligned}
& \frac{\partial \mathbf{v}_{N}^{\mathrm{I}}}{\partial t}=A^{\mathrm{I}} \frac{\partial \mathbf{v}_{N}^{\mathrm{I}}}{\partial x}+\tau^{\mathrm{I}}(t) T_{N+1}^{\prime}(x) \\
& \frac{\partial \mathbf{v}_{N}^{\mathrm{II}}}{\partial t}=A^{\mathrm{II}} \frac{\partial \mathbf{v}_{N}^{\mathrm{II}}}{\partial x}+\tau^{\mathrm{II}}(t) T_{N+1}^{\prime}(x) .
\end{aligned}
$$

Further, we prescribe initial conditions as before:

$$
\mathbf{v}(x, 0)=E \mathbf{u}(x, 0),
$$

where $E$ is the same projection operator.

Next, we regularize the boundary conditions. Let $F_{\varepsilon}(t)$ denote the approximate identity defined by

$$
F_{\varepsilon}(t)= \begin{cases}0 & \text { for } t<0 \\ \frac{e^{-t / \varepsilon}}{\varepsilon} & \text { for } t \geq 0\end{cases}
$$

Then our regularized boundary conditions are

$$
\begin{aligned}
& \mathbf{v}^{\mathrm{I}}(-1, t)=L\left(\left(\mathbf{v}^{\mathrm{II}}(-1) * F_{\varepsilon}\right)(t)\right)+\mathbf{g}^{\mathrm{I}}(t), \quad t \geq 0 \\
& \mathbf{v}^{\mathrm{II}}(1, t)=R\left(\left(\mathbf{v}^{\mathrm{I}}(1) * F_{\varepsilon}\right)(t)\right)+\mathbf{g}^{\mathrm{II}}(t),
\end{aligned}
$$

Here $*$ denotes the convolution operator

$$
(a * b)(t)=\int_{0}^{\infty} a(t-y) b(y) d y
$$

Note that with our modified boundary conditions, causality still holds-i.e., the present depends on the past but not on the future. In general, we could have chosen $F_{\varepsilon}(t)$ as any of a host of approximate identities, which in many respects have more desirable properties. However, we choose $F_{\varepsilon}(t)$ in the form above for simplicity of exposition.

In order to prove the stability and convergence of the modified spectral approximation (1.3) to the solution $\mathbf{u}(x, t)$ of the IBVP (1.1), we define an auxiliary initialboundary value problem for the hyperbolic system of partial differential equations (1.1a).

Let $\mathbf{w}(x, t, \varepsilon)$ denote the solution to the hyperbolic system of partial differential equations

$$
\frac{\partial \mathbf{w}^{\mathrm{I}}}{\partial t}=A^{\mathrm{I}} \frac{\partial \mathbf{w}^{\mathrm{I}}}{\partial x}, \quad \frac{\partial \mathbf{w}^{\mathrm{II}}}{\partial t}=A^{\mathrm{II}} \frac{\partial \mathbf{w}^{\mathrm{II}}}{\partial x},
$$

where $\mathbf{w}=\left(\mathbf{w}^{\mathrm{I}}, \mathbf{w}^{\mathrm{II}}\right)^{T}$. We prescribe initial conditions

$$
\mathbf{w}(x, 0)=\mathbf{f}(x)
$$

and boundary conditions

$$
\begin{aligned}
& \mathbf{w}^{\mathrm{I}}(-1, t)=L\left(\left(\mathbf{w}^{\mathrm{II}}(-1) * F_{\varepsilon}\right)(t)\right)+\mathbf{g}^{\mathrm{I}}(t), \quad t \geq 0 . \\
& \mathbf{w}^{\mathrm{II}}(1, t)=R\left(\left(\mathbf{w}^{\mathrm{I}}(1) * F_{\varepsilon}\right)(t)\right)+\mathbf{g}^{\mathrm{II}}(t),
\end{aligned}
$$

We then prove stability and the convergence of the modified spectral approximation (1.3) to the solution $\mathbf{u}(x, t)$ of $(1.1)$ in three steps:

(i) In Section 2 we prove that the modified spectral approximation (1.3) with zero initial conditions is stable. 
(ii) In Section 3 we show that the solution $\mathbf{w}(x, t, \varepsilon)$ of the auxiliary IBVP (1.4) converges to $\mathbf{u}(x, t)$ as $\varepsilon \rightarrow 0$, and we estimate the rate of convergence.

(iii) In Section 4 we prove that the solution $\mathbf{v}_{N}(x, t, \varepsilon)$ of the modified spectral approximation (1.3) converges to the solution $\mathbf{u}(x, t)$ of (1.1) if we first let $\varepsilon \rightarrow 0$, and then let $N \rightarrow \infty$. Our proof relies on the stability of the modified spectral approximation (1.3) which we establish in Section 2.

In another paper we shall present the results obtained from implementing the numerical scheme we propose in this paper, and also examine the optimal choice of the approximate identity employed in the method.

2. Stability of the Modified Spectral Method. In discussing the stability of the numerical scheme, a closely related question concerns the conditions under which the hyperbolic IBVP itself is stable.

To establish the well-posedness of (1.1), we must establish the following inequality with some $\eta_{0} \geq 0$ :

$$
\eta \int_{0}^{\infty} e^{-2 \eta t}\|\mathbf{u}(x, t)\|^{2} d t \leq \mathrm{const} \int_{0}^{\infty} e^{-2 \eta t}|\mathbf{g}(t)|^{2} d t
$$

for all $\eta>\eta_{0}$. Here,

$$
\|\mathbf{u}(x, t)\|^{2}=\int_{-1}^{1}|\mathbf{u}(x, t)|^{2} d x
$$

is the spatial norm of the vector-valued function $\mathbf{u}(x, t)$.

The parameter $\eta_{0}$ measures the exponential time growth of the solution.

It can be shown that for the problem under consideration, the value of $\eta_{0}$ is given by

$$
\eta_{0}>\max \{0,(\log (|L| \cdot|R|) \cdot|A|) / 4\} .
$$

We now return to the stability of the modified spectral approximation (1.3).

Definition. The approximation $(1.3 \mathrm{a})-(1.3 \mathrm{c})$ is stable if there exists a weighting pair $\omega(x)=\left(\omega^{\mathrm{I}}(x), \omega^{\mathrm{II}}(x)\right)$ and constants $\alpha$ and $\eta_{0}$, and an integer $N_{0}$, such that for all $\eta>\eta_{0}$ and $N \geq N_{0}$ we have

$$
\eta \int_{0}^{\infty} e^{-2 \eta t}\left\|\mathbf{v}_{N}(x, t)\right\|^{2} d t \leq \operatorname{const} N^{2 \alpha} \int_{0}^{\infty} e^{-2 \eta t}|\mathbf{g}(t)|^{2} d t
$$

where

$$
\begin{aligned}
\left\|\mathbf{v}_{N}(x, t)\right\|^{2} \equiv\left\|\mathbf{v}_{N}(x, t)\right\|_{\omega}^{2}= & \int_{-1}^{1}\left|\mathbf{v}_{N}^{\mathrm{I}}(x, t)\right|^{2} \omega^{\mathrm{I}}(x) d x \\
& +\int_{-1}^{1}\left|\mathbf{v}_{N}^{\mathrm{II}}(x, t)\right|^{2} \omega^{\mathrm{II}}(x) d x
\end{aligned}
$$

For spectral methods using Chebyshev polynomials, we choose

$$
\omega^{\mathrm{I}}(x)=(1-x) /\left(1-x^{2}\right)^{1 / 2} \quad \text { and } \quad \omega^{\mathrm{II}}(x)=(1+x) /\left(1-x^{2}\right)^{1 / 2} .
$$

We now state the main theorem of this section.

THEOREM 1. The modified pseudospectral approximation (1.3) is stable.

To prove this, we first look at the solution of the scalar problem

$$
\frac{\partial p_{N}}{\partial t}=a \frac{\partial p_{N}}{\partial x}+\alpha(t) T_{N+1}^{\prime}(x)
$$


and zero initial data. We need to introduce some notation. Let $s=\eta+i \xi$. Let $\hat{h}(s)$ denote the Laplace transform of $h(t)$,

$$
\hat{h}(s)=\int_{0}^{\infty} e^{-s t} h(t) d t .
$$

We have assumed in this definition that

$$
h(t)=0 \quad \text { for } t<0 .
$$

Taking the Laplace transform of (2.3), we get

$$
s \hat{p}_{N}(x, s)=a \frac{d}{d x} \hat{p}_{N}(x, s)+\hat{\alpha}(s) T_{N+1}^{\prime}(x) .
$$

This leads us to Theorem 5.2 of [1]:

THEOREM 5.2. Let $\hat{p}_{N}^{\mathrm{I}}(x, s)$ be a polynomial in $x$ of degree $N$ which satisfies the scalar inflow problem

$$
s \hat{p}_{N}^{\mathrm{I}}(x, s)=a \frac{d}{d x} \hat{p}_{N}^{\mathrm{I}}(x, s)+T_{N+1}^{\prime}(x), \quad a<0 .
$$

If $\hat{p}_{N}^{\mathrm{I}}\left(-1, s_{0}\right)=0$, then $\operatorname{Re} s_{0}<0$. In fact, $\hat{p}_{N}^{\mathrm{I}}(x, s)$ satisfies

$$
\left|\hat{p}_{N}^{\mathrm{I}}(1, s)\right| \leq\left|\hat{p}_{N}^{\mathrm{I}}(-1, s)\right|
$$

for $s$ such that $\operatorname{Re} s=\eta>0$.

Similarly, in the outflow scalar case:

$$
s \hat{p}_{N}^{\mathrm{II}}(x, s)=a \frac{d}{d x} \hat{p}_{N}^{\mathrm{II}}(x, s)+T_{N+1}^{\prime}(x), \quad a>0,
$$

if $\hat{p}_{N}^{\mathrm{II}}\left(1, s_{0}\right)=0$, then $\operatorname{Re} s_{0}<0$, and we have

$$
\left|\hat{p}_{N}^{\mathrm{II}}(1, s)\right| \geq\left|\hat{p}_{N}^{\mathrm{II}}(-1, s)\right|
$$

for $s$ such that $\operatorname{Re} s=\eta>0$.

Now we take the Laplace transform of (1.3) with respect to $t$ to obtain

$$
\begin{aligned}
& s \hat{\mathbf{v}}_{N}=A \frac{d \hat{\mathbf{v}}_{N}}{d x}+\hat{\tau}(s) T_{N+1}^{\prime}(x), \\
& \hat{\mathbf{v}}_{N}^{\mathrm{I}}(-1, s)=\frac{L}{(1+\varepsilon s)} \hat{\mathbf{v}}_{N}^{\mathrm{II}}(-1, s)+\hat{\mathbf{g}}^{\mathrm{I}}(s), \\
& \hat{\mathbf{v}}_{N}^{\mathrm{II}}(1, s)=\frac{R}{(1+\varepsilon s)} \hat{\mathbf{v}}_{N}^{\mathrm{I}}(1, s)+\hat{\mathbf{g}}^{\mathrm{II}}(s),
\end{aligned}
$$

since the Laplace transform $\hat{F}_{\varepsilon}(s)$ of $F_{\varepsilon}(t)$ is

$$
\hat{F}_{\varepsilon}(s)=1 /(1+\varepsilon s) \text {. }
$$

Let $P_{N}^{\mathrm{I}}(x, s)=p_{N}^{\mathrm{I}}\left(x, s, A^{\mathrm{I}}\right)$ and $P_{N}^{\mathrm{II}}(x, s)=p_{N}^{\mathrm{II}}\left(x, s, A^{\mathrm{II}}\right)$ denote the diagonal matrices

$$
\begin{aligned}
& P_{N}^{\mathrm{I}}(x, s)=\sum_{k=0}^{N} s^{-k-1}\left(T_{N+1}^{\prime}(x)\right)^{(k)}\left(A^{\mathrm{I}}\right)^{k} \\
& P_{N}^{\mathrm{II}}(x, s)=\sum_{k=0}^{N} s^{-k-1}\left(T_{N+1}^{\prime}(x)\right)^{(k)}\left(A^{\mathrm{II}}\right)^{k} .
\end{aligned}
$$


Then the solution of (2.7) is given by

$$
\hat{\mathbf{v}}_{N}^{\mathrm{I}}(x, s)=P_{N}^{\mathrm{I}}(x, s) \hat{\boldsymbol{\tau}}^{\mathrm{I}}, \quad \hat{\mathbf{v}}_{N}^{\mathrm{II}}(x, s)=P_{N}^{\mathrm{II}}(x, s) \hat{\boldsymbol{\tau}}^{\mathrm{II}},
$$

where $\hat{\tau}^{\mathrm{I}}$ and $\hat{\tau}^{\mathrm{II}}$ satisfy the linear system

$$
\left[\begin{array}{cc}
P_{N}^{\mathrm{I}}(-1, s) & \frac{-L P_{N}^{\mathrm{II}}(-1, s)}{(1+\varepsilon s)} \\
\frac{-R P_{N}^{\mathrm{I}}(1, s)}{(1+\varepsilon s)} & P_{N}^{\mathrm{II}}(1, s)
\end{array}\right]\left[\begin{array}{c}
\hat{\boldsymbol{\tau}}^{\mathrm{I}}(s) \\
\hat{\boldsymbol{\tau}}^{\mathrm{II}}(s)
\end{array}\right]=\left[\begin{array}{c}
\hat{\mathbf{g}}^{\mathrm{I}}(s) \\
\hat{\mathbf{g}}^{\mathrm{II}}(s)
\end{array}\right] .
$$

Let $\delta$ be a positive constant less than one. Choose

$$
\eta_{0}>\max \{0,(\log (|L| \cdot|R| /(1-\delta)) \cdot|A| / 4)\} .
$$

LEMMA 1. There exists an integer $N_{0}(\varepsilon)$ such that for all $s$ with $\operatorname{Re} s=\eta>\eta_{0}$ and all $N \geq N_{0}(\varepsilon)$ there holds

$$
\operatorname{det} D_{N}(s) \neq 0
$$

where

$$
D_{N}(s)=\left[\begin{array}{cc}
P_{N}^{\mathrm{I}}(-1, s) & \frac{-L P_{N}^{\mathrm{II}}(-1, s)}{(1+\varepsilon s)} \\
\frac{-R P_{N}^{\mathrm{I}}(1, s)}{(1+\varepsilon s)} & P_{N}^{\mathrm{II}}(1, s)
\end{array}\right]
$$

Here,

$$
\begin{aligned}
D_{N}(s) & =\left[\begin{array}{cc}
I & \frac{-L P_{N}^{\mathrm{II}}(-1, s)}{(1+\varepsilon s) P_{N}^{\mathrm{II}}(1, s)} \\
\frac{-R P_{N}^{\mathrm{I}}(1, s)}{(1+\varepsilon s) P_{N}^{\mathrm{I}}(-1, s)} & I
\end{array}\right]\left[\begin{array}{cc}
P_{N}^{\mathrm{I}}(-1, s) & 0 \\
0 & P_{N}^{\mathrm{II}}(1, s)
\end{array}\right] \\
& =E_{N}(s) \cdot P_{N}(s) .
\end{aligned}
$$

To prove the lemma, we examine the behavior of the family of functions

$$
\phi_{N}(s)=\frac{P_{N}^{\mathrm{II}}(-1, s)}{P_{N}^{\mathrm{II}}(1, s)}
$$

as $N \rightarrow \infty$. (Here and below, the ratio of two diagonal matrices is meant to be the product of the numerator matrix and the inverse of the denominator matrix.)

This leads us to

LEMMA 2. The family of diagonal matrices $\phi_{N}(s)$ converge uniformly on every compact subset of $\operatorname{Re} s>0$ to

$$
\phi(s)=e^{-2\left(A^{\mathrm{Il}}\right)^{-1} s} .
$$

Proof. By Theorem 5.2 of [1] we have that $\phi_{N}(s)$ is analytic and $\left|\phi_{N}(s)\right| \leq 1$ for $\operatorname{Re} s>0$, for all $N$. Hence $\left\{\phi_{N}(s)\right\}_{N=1}^{\infty}$ is a normal family of functions, i.e., for any sequence of $\phi_{N}(s)$ there is a subsequence that converges. Now

$$
\phi_{N}(s)=\frac{\sum_{k=0}^{N}(s)^{-k-1} T_{N+1}^{(k+1)}(-1)\left(A^{\mathrm{II}}\right)^{k}}{\sum_{k=0}^{N}(s)^{-k-1} T_{N+1}^{(k+1)}(1)\left(A^{\mathrm{II}}\right)^{k}} .
$$


Alternatively,

$$
\phi_{N}(s)=\frac{\left\{\sum_{k=0}^{N}\left(T_{N+1}^{(N+1-k)}(-1) / T_{N+1}^{(N+1)}(-1)\right) s^{k}\left(A^{\mathrm{II}}\right)^{-k}\right\}\left(A^{\mathrm{II}}\right)^{N}}{\left\{\sum_{k=0}^{N}\left(T_{N+1}^{(N+1-k)}(1) / T_{N+1}^{(N+1)}(1)\right) s^{k}\left(A^{\mathrm{II}}\right)^{-k}\right\}\left(A^{\mathrm{II}}\right)^{N}}
$$

We know that (see [3, p. 159, formula (A.6)]

$$
T_{N+1}^{(k)}(1)=\prod_{l=0}^{k-1}\left((N+1)^{2}-l^{2}\right) /(2 l+1) .
$$

Hence,

$$
\left.\frac{T_{N+1}^{(N+1-k)}(1)}{T_{N+1}^{(N+1)}(1)}=\left[\prod_{l=N-k+1}^{N}\left((N+1)^{2}-l^{2}\right)\right) /(2 l+1)\right]^{-1} .
$$

Fixing $k$ and letting $N \rightarrow \infty$, we obtain

$$
\lim _{N \rightarrow \infty}\left(\frac{T_{N+1}^{(N+1-k)}(1)}{T_{N+1}^{(N+1)}(1)}\right)=\lim _{N \rightarrow \infty}\left(\prod_{l=1}^{k} \frac{l(2(N+1)-l)}{2(N+1-l)+1}\right)^{-1}=\frac{1}{k !} .
$$

Similarly,

$$
\lim _{N \rightarrow \infty}\left(\frac{T_{N+1}^{(N+1-k)}(-1)}{T_{N+1}^{(N+1)}(-1)}\right)=\frac{(-1)^{k}}{k !} .
$$

We now restrict ourselves to $s=\eta$, where $\eta$ is real and $0<\eta<1 /\left(2 \cdot\left|A^{-1}\right|\right)$. Then it is easy to see that

$$
\begin{aligned}
\lim _{N \rightarrow \infty} \phi_{N}(\eta) & =\frac{\left\{\sum_{k=0}^{N}\left(T_{N+1}^{(N+1-k)}(-1) / T_{N+1}^{(N+1)}(-1)\right) \eta^{k}\left(A^{\mathrm{II}}\right)^{-k}\right\}}{\left\{\sum_{k=0}^{N}\left(T_{N+1}^{(N+1-k)}(1) / T_{N+1}^{(N+1)}(1)\right) \eta^{k}\left(A^{\mathrm{II}}\right)^{-k}\right\}} \\
& =\frac{\left\{\sum_{k=0}^{m}(-\eta)^{k}\left(A^{\mathrm{II}}\right)^{-k} / k !+O\left(\eta^{m+1}\right)\right\}}{\left\{\sum_{k=0}^{m}(\eta)^{k}\left(A^{\mathrm{II}}\right)^{-k} / k !+O\left(\eta^{m+1}\right)\right\}},
\end{aligned}
$$

where $m$ is arbitrary. Letting $m \rightarrow \infty$ we obtain

$$
\lim _{N \rightarrow \infty} \phi_{N}(\eta)=\frac{e^{-\left(A^{\mathrm{II}}\right)^{-1} \eta}}{e^{\left(A^{\mathrm{II}}\right)^{-1} \eta}}=e^{-2\left(A^{\mathrm{II}}\right)^{-1} \eta} \quad \text { for } 0<\eta<\frac{1}{2\left|A^{-1}\right|} .
$$

Now by Vitali's Theorem, if a normal family of functions $\left\{\phi_{N}(s)\right\}_{N=1}^{\infty}$ which are analytic in a domain $\Omega$ converges on a set of points in $\Omega$ which has a limit point in $\Omega$ to a function $\phi(s)$, then $\phi_{N}(s)$ converges uniformly to $\phi(s)$ on every compact set of $\Omega$. Hence, Lemma 2 follows.

Next, let

$$
\theta_{N}(s)=\frac{P_{N}^{\mathrm{I}}(1, s)}{P_{N}^{\mathrm{I}}(-1, s)}=\frac{\sum_{k=0}^{N}(s)^{-k-1} T_{N+1}^{(k+1)}(1)\left(A^{\mathrm{I}}\right)^{k}}{\sum_{k=0}^{N}(s)^{-k-1} T_{N+1}^{(k+1)}(-1)\left(A^{\mathrm{I}}\right)^{k}} .
$$

We can prove, as we did in Lemma 2, that $\theta_{N}(s)$ converges uniformly on every compact subset of $\operatorname{Re} s>0$ to

$$
\theta(s)=e^{2\left(A^{1}\right)^{-1} s}
$$


Now by (2.12),

$$
D_{N}(s)=E_{N}(s) \cdot P_{N}(s) .
$$

By Theorem 5.2 of [1] we know that $P_{N}(s)$ is invertible; so to prove that $\operatorname{det} D_{N}(s)$ $\neq 0$ we need to show that $E_{N}(s)$ is invertible. We may write $E_{N}(s)$ as

$$
E_{N}(s)=\left[\begin{array}{cc}
I & -\mathscr{L}_{N} \\
-\mathscr{R}_{N} & I
\end{array}\right]
$$

where

$$
\mathscr{L}_{N}(s)=\frac{L \phi_{N}(s)}{(1+\varepsilon s)} \quad \text { and } \quad \mathscr{R}_{N}(s)=\frac{R \theta_{N}(s)}{(1+\varepsilon s)} .
$$

Choose $d$ so large that for all $s$ with $|s| \geq d$ we have

$$
|L| \cdot|R| /|1+\varepsilon s|^{2}<1-\delta
$$

Let $K$ be the compact set

$$
K=\left\{s: \operatorname{Re} s=\eta \geq \eta_{0} \text { and }|s| \leq d\right\} .
$$

Then by Lemma $2, \phi_{N}(s) \cdot \theta_{N}(s)$ converges uniformly on $K$ to

$$
\psi(s)=e^{-2\left(A^{\mathrm{II}}\right)^{-1} s} \cdot e^{2\left(A^{\mathrm{I}}\right)^{-1} s} .
$$

Hence, we can find an integer $N_{0}$ such that for all $N \geq N_{0}$

$$
\left|\phi_{N}(s)\right| \cdot\left|\theta_{N}(s)\right|<\frac{1-\delta}{|L||R|} \quad \text { for all } s \in K \text {. }
$$

Now

$$
\begin{aligned}
\left|\left(I-\mathscr{R}_{N} \mathscr{L}_{N}\right)^{-1}\right| & \leq \frac{1}{1-\left|\mathscr{R}_{N}\right| \cdot\left|\mathscr{L}_{N}\right|} \\
& \leq \frac{1}{\left(1-|R| \cdot|L| \cdot\left|\phi_{N}(s) \cdot \theta_{N}(s)\right|\right) /|1+\varepsilon s|^{2}} \\
& \leq \frac{1}{\delta} \quad \text { for all } s \in K \text { and for all } N \geq N_{0} .
\end{aligned}
$$

Similarly, $\left|\left(I-\mathscr{L}_{N} \mathscr{R}_{N}\right)^{-1}\right| \leq 1 / \delta$ for all $s \in K$ and for all $N \geq N_{0}$. Next, consider the set $C=\left\{s:|s| \geq d\right.$ and $\left.\operatorname{Re} s \geq \eta_{0}\right\}$. Then, because of our choice of $d$,

$$
\begin{aligned}
\left|\left(I-\mathscr{R}_{N} \mathscr{L}_{N}\right)^{-1}\right| & \leq \frac{1}{1-\left|\mathscr{R}_{N}\right| \cdot\left|\mathscr{L}_{N}\right|} \\
& \leq \frac{1}{\left(1-|R| \cdot|L| \cdot\left|\phi_{N}(s) \cdot \theta_{N}(s)\right|\right) /|1+\varepsilon s|^{2}} \\
& \leq \frac{1}{\delta} \quad \text { for all } s \in C .
\end{aligned}
$$

Similarly, $\left|\left(I-\mathscr{L}_{N} \mathscr{R}_{N}\right)^{-1}\right| \leq 1 / \delta$ for all $s \in C$. Combining these two results, we conclude that for all $N \geq N_{0}$ and for all $s$ with $\operatorname{Re} s=\eta \geq \eta_{0}$,

$$
\left|\left(I-\mathscr{L}_{N} \mathscr{R}_{N}\right)^{-1}\right|,\left|\left(1-\mathscr{R}_{N} \mathscr{L}_{N}\right)^{-1}\right| \leq 1 / \delta \text {. }
$$

Hence, $E_{N}(s)$ can be inverted:

$$
E_{N}^{-1}(s)=\left[\begin{array}{cc}
I & \mathscr{R}_{N} \\
\mathscr{L}_{N} & I
\end{array}\right]\left[\begin{array}{cc}
\left(I-\mathscr{R}_{N} \mathscr{L}_{N}\right)^{-1} & 0 \\
0 & \left(I-\mathscr{L}_{N} \mathscr{R}_{N}\right)^{-1}
\end{array}\right],
$$


and the $\varepsilon$-independent estimate

$$
\left|E_{N}^{-1}(s)\right| \leq\left(2+|R|^{2}+|L|^{2}\right)^{1 / 2} \cdot \frac{1}{\delta} \leq \frac{1+|R|+|L|}{\delta}
$$

follows, for any $\varepsilon>0$ and all $N \geq N_{0}(\varepsilon)$. This completes the proof of Lemma 1.

We can now prove Theorem 1 .

System (1.3) consists of $l$ inflow equations

$$
s \hat{v}_{N}^{j}=a_{j} \frac{d}{d x} \hat{v}_{N}^{j}+\hat{\tau}^{j} T_{N-1}^{\prime}(x), \quad 1 \leq j \leq l,
$$

and $(n-l)$ outflow equations

$$
s \hat{v}_{N}^{j}=a_{j} \frac{d}{d x} \hat{v}_{N}^{j}+\hat{\tau}^{j} T_{N+1}^{\prime}(x), \quad l+1 \leq j \leq n .
$$

By Theorem (5.1) of [1] there exists a weighting pair $\omega(x)=\left(\omega^{\mathrm{I}}(x), \omega^{\mathrm{II}}(x)\right)$ and constants $\alpha$ and $\eta_{0} \geq 0$ such that for all $s$ with $\operatorname{Re} s=\eta>\eta_{0}$ we have for arbitrary vectors $\tau$

$$
\begin{aligned}
& \eta\left\|\hat{v}_{N}^{j}(x, s)\right\|_{\omega^{1}}^{2} \leq \text { const } N^{2 \alpha}\left|\hat{v}_{N}^{j}(-1, s)\right|^{2}, \quad 1 \leq j \leq l, \\
& \eta\left\|\hat{v}_{N}^{j}(x, s)\right\|_{\omega^{11}}^{2} \leq \text { const } N^{2 \alpha}\left|\hat{v}_{N}^{j}(1, s)\right|^{2}, \quad l+1 \leq j \leq n .
\end{aligned}
$$

Using the spatial norm

$$
\left\|\hat{\mathbf{v}}_{N}(x, s)\right\|_{\omega}^{2}=\left\|\hat{\mathbf{v}}_{N}^{\mathrm{I}}(x, s)\right\|_{\omega^{\mathrm{I}}}^{2}+\left\|\hat{\mathbf{v}}_{N}^{\mathrm{II}}(x, s)\right\|_{\omega^{\mathrm{II}}}^{2},
$$

these inequalities can be added together and rewritten in concise form as

$$
\eta\left\|\hat{\mathbf{v}}_{N}(x, s)\right\|_{\omega}^{2} \leq \mathrm{const} N^{2 \alpha}\left[\left|\hat{\mathbf{v}}_{N}^{\mathrm{I}}(-1, s)\right|^{2}+\left|\hat{\mathbf{v}}_{N}^{\mathrm{II}}(1, s)\right|^{2}\right]
$$

for $\operatorname{Re} s=\eta>\eta_{0}$. We now have to estimate the boundary terms on the right. We have

$$
\hat{\mathbf{v}}_{N}^{\mathrm{I}}(x, s)=P_{N}^{\mathrm{I}}(x, s) \hat{\boldsymbol{\tau}}^{\mathrm{I}}, \quad \hat{\mathbf{v}}_{N}^{\mathrm{II}}(x, s)=P_{N}^{\mathrm{II}}(x, s) \hat{\boldsymbol{\tau}}^{\mathrm{II}} .
$$

Inserting these relations into the boundary conditions, we get

$$
\left[\begin{array}{c}
\hat{\boldsymbol{\tau}}^{\mathrm{I}}(s) \\
\hat{\boldsymbol{\tau}}^{\mathrm{II}}(s)
\end{array}\right]=D_{N}^{-1}(s)\left[\begin{array}{c}
\hat{\mathbf{g}}^{\mathrm{I}}(s) \\
\hat{\mathbf{g}}^{\mathrm{II}}(s)
\end{array}\right] .
$$

Furthermore,

$$
\left[\begin{array}{c}
\hat{\mathbf{v}}_{N}^{\mathrm{I}}(-1, s) \\
\hat{\mathbf{v}}_{N}^{\mathrm{II}}(1, s)
\end{array}\right]=\left[\begin{array}{cc}
P_{N}^{\mathrm{I}}(-1, s) & 0 \\
0 & P_{N}^{\mathrm{II}}(1, s)
\end{array}\right]\left[\begin{array}{c}
\hat{\boldsymbol{\tau}}^{\mathrm{I}}(s) \\
\hat{\boldsymbol{\tau}}^{\mathrm{II}}(s)
\end{array}\right] .
$$

So we conclude

$$
\left[\begin{array}{c}
\hat{\mathbf{v}}_{N}^{\mathrm{I}}(-1, s) \\
\hat{\mathbf{v}}_{N}^{\mathrm{II}}(1, s)
\end{array}\right]=E_{N}^{-1}(s)\left[\begin{array}{c}
\hat{\mathbf{g}}^{\mathrm{I}}(s) \\
\hat{\mathbf{g}}^{\mathrm{II}}(s)
\end{array}\right]
$$

Since by Lemma $2, E_{N}(s)$ has a uniformly bounded inverse for Re $s=\eta \geq \eta_{0}$ and $N \geq N_{0}$, we get

$$
\left|\hat{\mathbf{v}}_{N}^{\mathrm{I}}(-1, s)\right|^{2}+\left|\hat{\mathbf{v}}_{N}^{\mathrm{II}}(1, s)\right|^{2} \leq K|\hat{\mathbf{g}}(s)|^{2} .
$$

Combining this with (2.13), we conclude

$$
\eta\left\|\hat{\mathbf{v}}_{N}(s, x)\right\|_{\omega}^{2} \leq \text { const } N^{2 \alpha}|\hat{\mathbf{g}}(s)|^{2} .
$$

This completes the proof of Theorem 1 . 
3. Convergence of the Modified IBVP. We prove that $\mathbf{w}(x, t, \varepsilon)$, the solution of the modified IBVP (1.4), converges to $\mathbf{u}(x, t)$, the solution of the original IBVP (1.1) as $\varepsilon \rightarrow 0$, and we estimate the rate of convergence.

In [6], Rauch has proved that the solution $\mathbf{u}(x, t)$ of (1.1) satisfies an estimate of the form

$$
\begin{aligned}
\eta \sum_{|\alpha| \leq m} & \int_{0}^{\infty} \int_{-1}^{1}\left|\partial_{x}^{\alpha_{1}} \partial_{t}^{\alpha_{2}} \mathbf{u}(x, t)\right|^{2} e^{-2 \eta t} d x d t \\
& +\sum_{\alpha \leq m} \int_{0}^{\infty}\left\{\left|\partial_{t}^{\alpha} \mathbf{u}(1, t)\right|^{2}+\left|\partial_{t}^{\alpha} \mathbf{u}(-1, t)\right|^{2}\right\} e^{-2 \eta t} d t \\
\leq & C_{m}\left(\sum_{\alpha \leq m}\left[\int_{-1}^{1}\left|\partial_{x}^{\alpha} \mathbf{f}(x)\right|^{2} d x+\int_{0}^{\infty}\left|\partial_{t}^{\alpha} \mathbf{g}(t)\right|^{2} e^{-2 \eta t} d t\right]\right)
\end{aligned}
$$

for all integers $m \geq 0$ and all $\eta>\eta_{0}$, with $C_{m}$ independent of $\mathbf{f}$ and $\mathbf{g}$.

In particular, estimate (3.1) yields the weaker inequality

$$
\begin{aligned}
& \sum_{\alpha \leq 1} \int_{0}^{\infty}\left\{\left|\partial_{t}^{\alpha} \mathbf{u}(1, t)\right|^{2}+\left|\partial_{t}^{\alpha} \mathbf{u}(-1, t)\right|^{2}\right\} e^{-2 \eta t} d t \\
& \quad \leq C_{1}\left(\sum_{\alpha \leq 1}\left[\int_{-1}^{1}\left|\partial_{x}^{\alpha} \mathbf{f}(x)\right|^{2} d x+\int_{0}^{\infty}\left|\partial_{t}^{\alpha} \mathbf{g}(t)\right|^{2} e^{-2 \eta t} d t\right]\right) .
\end{aligned}
$$

Now consider the modified IBVP (1.4). It is easy to show that if we prescribe zero initial data for the problem, i.e., if we solve

$$
\begin{aligned}
& \frac{\partial \mathrm{l}}{\partial t}=A \frac{\partial \mathrm{l}}{\partial x} \\
& \mathrm{l}(x, 0)=0 \\
& \mathbf{l}^{\mathrm{I}}(-1, t)=L\left(\left(\mathrm{l}^{\mathrm{II}}(-1) * F_{\varepsilon}\right)(t)\right)+\mathbf{g}^{\mathrm{I}}(t), \\
& \mathrm{l}^{\mathrm{II}}(1, t)=R\left(\left(\mathbf{l}^{\mathrm{I}}(1) * F_{\varepsilon}\right)(t)\right)+\mathrm{g}^{\mathrm{II}}(t),
\end{aligned}
$$

then the solution $\mathrm{l}(x, t)$ satisfies the estimate

$$
\eta \int_{0}^{\infty} e^{-2 \eta t}\|\mathbf{l}(x, t)\|^{2} d t \leq \mathrm{const} \int_{0}^{\infty} e^{-2 \eta t}|\mathbf{g}(t)|^{2} d t
$$

for all $\eta>\eta_{0}$. We sketch the proof.

Taking the Laplace transform of (3.3), we obtain

$$
\begin{aligned}
& s \hat{\mathbf{l}}=A \frac{d \hat{\mathbf{l}}}{d x}, \\
& \hat{\mathbf{l}}^{\mathrm{I}}(-1, s)=\frac{L}{(1+\varepsilon s)} \hat{\mathbf{l}}^{\mathrm{II}}(-1, s)+\hat{\mathbf{g}}^{\mathrm{I}}(s), \\
& \hat{\mathbf{l}}^{\mathrm{II}}(1, s)=\frac{R}{(1+\varepsilon s)} \hat{\mathbf{l}}^{\mathrm{I}}(1, s)+\hat{\mathrm{g}}^{\mathrm{II}}(s) .
\end{aligned}
$$

The solution of (3.5) is

$$
\begin{aligned}
& \hat{\mathbf{l}}^{\mathrm{I}}(x, s)=e^{s\left(A^{\mathrm{I}}\right)^{-1}(x+1)} \lambda^{\mathrm{I}}(s), \\
& \hat{\mathbf{l}}^{\mathrm{II}}(x, s)=e^{s\left(A^{\mathrm{II}}\right)^{-1}(x-1)} \lambda^{\mathrm{II}}(s),
\end{aligned}
$$


where $\lambda(s)=\left(\lambda^{\mathrm{I}}(s), \lambda^{\mathrm{II}}(s)\right)^{T}$ satisfies the linear system

Here,

$$
X(s) \lambda(s)=\hat{\mathbf{g}}(s) .
$$

$$
X(s)=\left[\begin{array}{cc}
I & \frac{-L e^{-2 s\left(A^{\mathrm{II}}\right)^{-1}}}{(1+\varepsilon s)} \\
\frac{-R e^{2 s\left(A^{\mathrm{l}}\right)^{-1}}}{(1+\varepsilon s)} & I
\end{array}\right] .
$$

It is easy to show, as in Section 2, that for $\operatorname{Re} s=\eta>\eta_{0}$ the inverse $X^{-1}(s)$ exists and satisfies the inequality

$$
\left|X^{-1}(s)\right| \leq K
$$

with $K$ a uniform constant. Thus we obtain

$$
|\lambda(s)| \leq K|\hat{\mathbf{g}}(s)| .
$$

Let $a=1 /|A|$. Then from (3.6) and the above inequality we conclude that

$$
\begin{aligned}
& \left|\hat{\mathbf{l}}^{\mathrm{I}}(x, s)\right| \leq K|\hat{\mathbf{g}}(s)| e^{-a \eta(1+x)}, \\
& \left|\hat{\mathbf{l}}^{\mathrm{II}}(x, s)\right| \leq K|\hat{\mathbf{g}}(s)| e^{-a \eta(1-x)} .
\end{aligned}
$$

By the Plancherel Theorem we have the identity

$$
\begin{aligned}
\int_{0}^{\infty}\|\mathbf{l}(x, t)\|_{\omega}^{2} e^{-2 \eta t} d t= & \int_{-1}^{1} \frac{(1-x)}{\left(1-x^{2}\right)^{1 / 2}} d x \int_{-\infty}^{\infty}\left|\hat{\mathbf{l}}^{\mathrm{I}}(x, \eta+i \xi)\right|^{2} d \xi \\
& +\int_{-1}^{1} \frac{(1+x)}{\left(1-x^{2}\right)^{1 / 2}} d x \int_{-\infty}^{\infty}\left|\hat{\mathbf{l}}^{\mathrm{II}}(x, \eta+i \xi)\right|^{2} d \xi
\end{aligned}
$$

and substituting the inequality (3.7) in the right-hand side, we obtain the estimate (3.4).

Now consider the modified IBVP (1.4), which is meant to approximate the original IBVP (1.1). Define

$$
\mathbf{e}(x, t, \varepsilon)=\mathbf{w}(x, t, \varepsilon)-\mathbf{u}(x, t) .
$$

Then $\mathbf{e}(x, t, \varepsilon)$ satisfies the modified IBVP with homogeneous initial conditions

$$
\begin{aligned}
& \frac{\partial \mathbf{e}}{\partial t}=A \frac{\partial \mathbf{e}}{\partial x}, \\
& \mathbf{e}(x, 0)=0, \\
& \mathbf{e}^{\mathrm{I}}(-1, t)=L\left(\left(\mathbf{e}^{\mathrm{II}}(-1) * F_{\varepsilon}\right)(t)\right)+\mathbf{h}^{\mathrm{I}}(t), \\
& \mathbf{e}^{\mathrm{II}}(1, t)=R\left(\left(\mathbf{e}^{\mathrm{I}}(1) * F_{\varepsilon}\right)(t)\right)+\mathbf{h}^{\mathrm{II}}(t),
\end{aligned}
$$

where

$$
\mathbf{h}^{\mathrm{I}}(t)=L\left(\left(\mathbf{u}^{\mathrm{II}}(-1) * F_{\varepsilon}\right)(t)-\mathbf{u}^{\mathrm{II}}(-1, t)\right)
$$

and

$$
\mathbf{h}^{\mathrm{II}}(t)=R\left(\left(\mathbf{u}^{\mathrm{I}}(1) * F_{\varepsilon}\right)(t)-\mathbf{u}^{\mathrm{I}}(1, t)\right) .
$$

Hence, by (3.4), we may conclude that the inequality

$$
\eta \int_{0}^{\infty} e^{-2 \eta t}\|\mathbf{e}(x, t)\|^{2} d t \leq \mathrm{const} \int_{0}^{\infty} e^{-2 \eta t}|\mathbf{h}(t)|^{2} d t
$$

holds for all $\eta>\eta_{0}$. 
Next, we estimate the right-hand side of (3.10). We have

$$
\begin{aligned}
\int_{0}^{\infty} & \left|\mathbf{h}^{\mathrm{I}}(t)\right|^{2} e^{-2 \eta t} d t \\
& \leq|L|^{2} \int_{0}^{\infty}\left|\mathbf{u}^{\mathrm{II}}(-1, t) * F_{\varepsilon}(t)-\mathbf{u}^{\mathrm{II}}(-1, t)\right|^{2} e^{-2 \eta t} d t \\
& \leq|L|^{2} \int_{0}^{\infty}|\varepsilon s /(1+\varepsilon s)|^{2}\left|\hat{\mathbf{u}}^{\mathrm{II}}(-1, \eta+i \xi)\right|^{2} d \xi \\
& \leq|L|^{2} \varepsilon^{2} \int_{0}^{\infty}|s|^{2}\left|\hat{\mathbf{u}}^{\mathrm{II}}(-1, \eta+i \xi)\right|^{2} d \xi
\end{aligned}
$$

Hence we conclude that

$$
\int_{0}^{\infty}\left|\mathbf{h}^{\mathrm{I}}(t)\right|^{2} e^{-2 \eta t} d t \leq \mathrm{const} \varepsilon^{2} \int_{0}^{\infty}\left|\partial_{t} \mathbf{u}^{\mathrm{II}}(-1, t)\right|^{2} e^{-2 \eta t} d t
$$

Similarly, we can show that

$$
\int_{0}^{\infty}\left|\mathbf{h}^{\mathrm{II}}(t)\right|^{2} e^{-2 \eta t} d t \leq \mathrm{const} \varepsilon^{2} \int_{0}^{\infty}\left|\partial_{t} \mathbf{u}^{\mathrm{I}}(1, t)\right|^{2} e^{-2 \eta t} d t .
$$

Combining these results, we obtain

$$
\begin{aligned}
& \int_{0}^{\infty}|h(t)|^{2} e^{-2 \eta t} d t \\
& \quad \leq \operatorname{const} \varepsilon^{2}\left(\int_{0}^{\infty}\left\{\left.\partial_{t} \mathbf{u}(1, t)\right|^{2}+\left|\partial_{t} \mathbf{u}(-1, t)\right|^{2}\right\} e^{-2 \eta t} d t\right.
\end{aligned}
$$

for all $\eta>\eta_{0}$.

Putting together the relations $(3.2),(3.10)$ and (3.11), we obtain the main estimate of this section:

$$
\begin{aligned}
\eta \int_{0}^{\infty} & \|\mathbf{u}(x, t)-\mathbf{w}(x, t, \varepsilon)\|_{\omega}^{2} e^{-2 \eta t} d t \\
& \leq \operatorname{const} \cdot \varepsilon^{2}\left(\sum_{\alpha \leq 1}\left[\int_{-1}^{1}\left|\partial_{x}^{\alpha} \mathbf{f}(x)\right|^{2} d x+\int_{0}^{\infty}\left|\partial_{t}^{\alpha} \mathbf{g}(t)\right|^{2} e^{-2 \eta t} d t\right]\right)
\end{aligned}
$$

for all $\eta>\eta_{0}$.

In particular, we have that

$$
\eta \int_{0}^{\infty}\|\mathbf{u}(x, t)-\mathbf{w}(x, t, \varepsilon)\|_{\omega}^{2} e^{-2 \eta t} d t=O\left(\varepsilon^{2}\right)
$$

for $\eta>\eta_{0}$.

4. Convergence of the Modified Spectral Approximation. In the preceding section we proved that $\mathbf{w}(x, t, \varepsilon)$, the solution of the modified IBVP (1.4), converges to $\mathbf{u}(x, t)$ linearly in $\varepsilon$ as $\varepsilon \rightarrow 0$.

Hence, to prove that $\mathbf{v}_{N}(x, t, \varepsilon)$, the solution of the modified spectral approximation (1.3), converges to $\mathbf{u}(x, t)$ as $\varepsilon \rightarrow 0$ and $N \rightarrow \infty$, it is enough to show that for fixed $\varepsilon, \mathbf{v}_{N}(x, t, \varepsilon) \rightarrow \mathbf{w}(x, t, \varepsilon)$ as $N \rightarrow \infty$.

The proof of this relies on the stability result we have established in Section 2, and is essentially the same as the proof of convergence in [2]. However, for the sake of completeness we shall provide a sketch of the proof. 
Let $\mathbf{r}$ be the solution of

$$
\begin{aligned}
& \frac{\partial \mathbf{r}}{\partial t}=A \frac{\partial \mathbf{r}}{\partial x} \\
& \mathbf{r}(x, 0)=\mathbf{f}(x)=\mathbf{w}(x, 0, \varepsilon) \\
& \mathbf{r}^{\mathrm{I}}(-1, t)=\mathbf{w}^{\mathrm{I}}(-1, t, \varepsilon), \\
& \mathbf{r}^{\mathrm{II}}(1, t)=\mathbf{w}^{\mathrm{II}}(1, t, \varepsilon)
\end{aligned}
$$

Then $\mathbf{r}(x, t) \equiv \mathbf{w}(x, t, \varepsilon)$.

Let $\mathbf{s}$ be the pseudospectral approximation,

$$
\begin{aligned}
& \frac{\partial \mathbf{s}}{\partial t}=A \frac{\partial \mathbf{s}}{\partial x}+T_{N+1}^{\prime}(x) \cdot \boldsymbol{\theta}(t), \\
& \mathbf{s}(x, 0)=E \mathbf{f}(x)=E \mathbf{w}(x, 0, \varepsilon), \\
& \mathbf{s}^{\mathrm{I}}(-1, t)=\mathbf{w}^{\mathrm{I}}(-1, t, \varepsilon), \\
& \mathbf{s}^{\mathrm{II}}(1, t)=\mathbf{w}^{\mathrm{II}}(1, t, \varepsilon) .
\end{aligned}
$$

Here, we define the projection operator $E=\left(E^{\mathrm{I}}, E^{\mathrm{II}}\right)$ by requiring that for any function $F, E^{\mathrm{I}} F$ and $E^{\mathrm{II}} F$ are polynomials of degree $N$ at most, satisfying

$$
\begin{array}{ll}
E^{\mathrm{I}} F\left(x_{j}\right)=F\left(x_{j}\right), & j=1, \ldots, N+1, \\
E^{\mathrm{II}} F\left(x_{j}\right)=F\left(x_{j}\right), & j=0, \ldots, N,
\end{array}
$$

where the points $x_{j}$ are defined by

$$
x_{j}=\cos (\pi j /(N+1)), \quad j=0, \ldots, N+1 .
$$

Then we define

$$
E \mathbf{f}=\left(E^{\mathrm{I}} \mathbf{f}^{\mathrm{I}}, E^{\mathrm{II}} \mathbf{f}^{\mathrm{II}}\right)^{T} .
$$

Let $\delta=\mathbf{s}-E \mathbf{r}$ and $\sigma=E \mathbf{r}-\mathbf{r}$. Then it is shown in [2] that for $\eta>0$ the following estimate holds:

$$
\begin{gathered}
\eta \int_{0}^{\infty} e^{-2 \eta t}\|\boldsymbol{\delta}(x, t)\|_{\omega}^{2} d t+\frac{1}{2 N} \int_{0}^{\infty} e^{-2 \eta t}\left|\delta^{\mathrm{I}}(-1, t)\right|^{2} d t \\
\quad+\frac{1}{2 N} \int_{0}^{\infty} e^{-2 \eta t}\left|\delta^{\mathrm{II}}(1, t)\right|^{2} d t \\
\leq \operatorname{const} \int_{0}^{\infty} e^{-2 \eta t}\|Q(x, t)\|^{2} d t
\end{gathered}
$$

where $Q=\left(Q^{\mathrm{I}}, Q^{\mathrm{II}}\right)^{T}$ is defined by

$$
Q^{\mathrm{I}}=A^{\mathrm{I}}\left(E^{\mathrm{I}} \frac{\partial \mathbf{w}^{\mathrm{I}}}{\partial x}-\frac{\partial}{\partial x} E^{\mathrm{I}} \mathbf{w}^{\mathrm{I}}\right), \quad Q^{\mathrm{II}}=A^{\mathrm{II}}\left(E^{\mathrm{II}} \frac{\partial \mathbf{w}^{\mathrm{II}}}{\partial x}-\frac{\partial}{\partial x} E^{\mathrm{II}} \mathbf{w}^{\mathrm{II}}\right)
$$

and

$$
\begin{aligned}
\|Q(x, t)\|^{2}= & \frac{\pi}{N} \sum_{j=1}^{N}\left(1-x_{j}\right)^{2}\left(1+x_{j}\right)\left|Q^{\mathrm{I}}\left(x_{j}, t\right)\right|^{2} \\
& +\frac{\pi}{N} \sum_{j=1}^{N}\left(1+x_{j}\right)^{2}\left(1-x_{j}\right)\left|Q^{\mathrm{II}}\left(x_{j}, t\right)\right|^{2}
\end{aligned}
$$


Next, we compare the modified spectral solution $\mathbf{s}$ with $\mathbf{v}$, as defined by (1.3). Then $\mathbf{v}-\mathbf{s}$ satisfies

$$
\begin{aligned}
& \frac{\partial}{\partial t}(\mathbf{v}-\mathbf{s})=\frac{\partial}{\partial x}(\mathbf{v}-\mathbf{s})+T_{N+1}^{\prime}(x) \cdot(\tau-\theta) \\
& (\mathbf{v}-\mathbf{s})(x, 0)=0 \\
& \left(\mathbf{v}^{\mathrm{I}}-\mathbf{s}^{\mathrm{I}}\right)(-1, t)=L\left(\left(\left(\mathbf{v}^{\mathrm{II}}-\mathbf{s}^{\mathrm{II}}\right)(-1) * F_{\varepsilon}\right)(t)\right)+\mathbf{b}^{\mathrm{I}}(t) \\
& \left(\mathbf{v}^{\mathrm{II}}-\mathbf{s}^{\mathrm{II}}\right)(1, t)=R\left(\left(\left(\mathbf{v}^{\mathrm{I}}-\mathbf{s}^{\mathrm{I}}\right)(1) * F_{\varepsilon}\right)(t)\right)+\mathbf{b}^{\mathrm{II}}(t)
\end{aligned}
$$

where

$$
\begin{aligned}
& \mathbf{b}^{\mathrm{I}}(t)=L\left(\left(\left(\delta^{\mathrm{II}}+\sigma^{\mathrm{II}}\right)(-1) * F_{\varepsilon}\right)(t)\right), \\
& \mathbf{b}^{\mathrm{II}}(t)=R\left(\left(\left(\delta^{\mathrm{I}}+\sigma^{\mathrm{I}}\right)(1) * F_{\varepsilon}\right)(t)\right) .
\end{aligned}
$$

By Theorem 1 of Section 2 we then have

$$
\eta \int_{0}^{\infty} e^{-2 \eta t}\|\mathbf{s}-\mathbf{v}\|_{\omega}^{2} d t \leq \operatorname{const} N^{2 \alpha} \int_{0}^{\infty} e^{-2 \eta t}|\mathbf{b}(t)|^{2} d t
$$

Clearly,

$$
\begin{aligned}
& \int_{0}^{\infty} e^{-2 \eta t}|\mathbf{b}(t)|^{2} d t \\
& \quad \leq \operatorname{const} \int_{0}^{\infty} e^{-2 \eta t}\left\{|\boldsymbol{\delta}(1, t)|^{2}+|\delta(-1, t)|^{2}+|\boldsymbol{\sigma}(1, t)|^{2}+|\boldsymbol{\sigma}(-1, t)|^{2}\right\} d t .
\end{aligned}
$$

Combining (4.3), (4.4) and (4.6), we obtain

$$
\begin{aligned}
& \eta \int_{0}^{\infty} e^{-2 \eta t}\|(\mathbf{s}-\mathbf{v})(x, t)\|^{2} d t \\
& \quad \leq \text { const } N^{2 \alpha+1} \int_{0}^{\infty} e^{-2 \eta t}\left\{\|Q(x, t)\|^{2}+|\boldsymbol{\sigma}(-1, t)|^{2}+|\boldsymbol{\sigma}(1, t)|^{2}\right\} d t .
\end{aligned}
$$

For smooth $\mathbf{w}(x, t, \varepsilon)$, the right-hand side tends to zero spectrally in $N$, i.e., it tends to zero faster than any power of $1 / N$.

Now

$$
\mathbf{w}-\mathbf{v}=(\mathbf{r}-E \mathbf{r})+(E \mathbf{r}-\mathbf{s})+(\mathbf{s}-\mathbf{v}) .
$$

Each of the three terms on the right tends to zero spectrally in $N$. This completes the proof of convergence.

Acknowledgments. The author would like to thank Dr. David Gottlieb for suggesting this problem and for his invaluable help and encouragement during the author's stay at ICASE.

Department of Mathematics Indian Institute of Technology Kanpur P. O.-I. I. T. Kanpur 208016 India

1. D. Gottlieb, L. Lustman \& E. Tadmor, Stability Analysis of Spectral Methods for Hyperbolic Initial Boundary Value Systems, NASA Contractor Report No. 178041, ICASE Report No. 86-2.

2. D. GotTlieb, L. Lustman \& E. TADMOR, Convergence of Spectral Methods for Hyperbolic Initial Boundary Value Systems, NASA Contractor Report No. 178063, ICASE Report No. 86-8. 
3. D. Gottlieb \& S. A. Orszag, Numerical Analysis of Spectral Methods: Theory and Applications, SIAM, Philadelphia, 1984.

4. H. O. KREISS, "Initial boundary value problems for hyperbolic systems," Comm. Pure Appl. Math., v. 23, 1970, pp. 277-298.

5. L. LUSTMAN, "The time evolution of spectral discretizations of hyperbolic systems," SIAM J. Numer. Anal. (To appear).

6. J. RAUCH, " $\mathfrak{L}_{2}$ is a continuable initial condition for Kreiss' mixed problems," Comm. Pure Appl. Math., v. 25, 1972, pp. 265-285. 